\title{
DERIVAÇÃO RETROESTERNAL COM TUBO GÁSTRICO ISOPERISTÁLTICO NO CARCINOMA IRRESSECÁVEL DE ESÔFAGO
}

\author{
RETROSTERNAL BYPASS OPERATION WITH ISOPERISTALTIC GASTRIC TUBE \\ FOR UNRESECTABLE ESOPHAGUS CARCINOMA
}

\author{
José Luís Braga de Aquino, TCBC - SP1 \\ Cirilo Luís Pardo Meo Muraro, TCBC - SP1 \\ José Gonzaga Teixeira de Camargo ${ }^{2}$ \\ Guilherme Otranto ${ }^{3}$ \\ Rogério Abreu ${ }^{3}$
}

\begin{abstract}
RESUMO: Objetivo: Avaliar o benefício do tratamento paliativo pela derivação esofágica com o tubo gástrico isoperistáltico em pacientes com carcinoma de esôfago irressecável. Método: Foram estudados 53 pacientes com carcinoma espino celular do esôfago sem condições de ressecabilidade avaliados por critérios endoscópicos e radiológicos. A maioria dos pacientes era do sexo masculino com idade média de 56,8 anos. A operação realizada foi a derivação esofágica com o tubo gástrico isoperistáltico, de grande curvatura e transposto através do espaço retro esternal. Resultados: Vinte e oito pacientes $(52,0 \%)$ desenvolveram uma ou mais complicações, sendo a mais freqüente a deiscência e/ou estenose da anastomose cervical (15 pacientes - 28,3\%). Em 48 pacientes que sobreviveram, 37 (77,0\%) referiram alívio da disfagia no seguimento pósoperatório. A média de sobrevida em 23 pacientes foi de sete meses e meio (seis a 13 meses) e 14 pacientes estão em seguimento com o tempo variável entre dois e 16 meses, com boa evolução, com perda de seguimento nos 11 pacientes restantes. Conclusões: Tubo gástrico isoperistáltico tem aceitável morbidade e mortalidade para a população em estudo, permitindo paliação da disfagia na maioria dos casos.
\end{abstract}

Descritores: Esôfago; Neoplasias esofágicas; Assistência Paliativa.

\section{INTRODUÇÃO}

A neoplasia de esôfago é doença muito comum em regiões com populações de baixa renda e aquelas habituadas à ingestão excessiva de álcool e abuso de tabaco ${ }^{1-4 .}$

Quase sempre, o diagnóstico desta afecção é tardio, pois a disfagia, principal sintoma da neoplasia de esôfago, ocorre somente quando mais de dois terços da luz esofágica está preenchida por massa tumoral.

O retardo no diagnóstico, a perda de peso excessiva consequiente à disfagia e a associação de doenças cardio-pulmonares decorrentes ${ }^{5}$ do abuso de tabaco, tornam o portador de neoplasia de esôfago paciente de difícil controle clínico, com poucas possibilidades terapêuticas. Em várias séries de trata-

1. Professor Titular de Clínica Cirúrgica da F.C.M. PUC - Campinas.

2. Professor Adjunto de Clínica Cirúrgica da F.C.M. PUC - Campinas.

3. Alunos do Internato de Clínica Cirúrgica da F.C.M. PUC - Campinas.

Recebido em: 26/03/2002

Aceito para publicação em: 11/02/2003

Trabalho realizado no Serviço de Clínica Cirúrgica do Hospital e Maternidade Celso Pierro da F.C.M. PUC - Campinas. 
mento da neoplasia de esôfago, principalmente do Mundo Ocidental, apenas cerca de 20 a $30 \%$ dos pacientes conseguem realizar tratamento cirúrgico curativo ${ }^{4-6}$

Durante a década de 80 e 90, preconizou-se o uso de próteses, quer introduzida por via endoscópica ou por via cirúrgica. Este método permite realimentação oral imediata, e impede a broncopneumonia aspirativa ${ }^{7,8}$. Entretanto, a morbidade não é desprezível, variando de 22 a $60 \%$ e o óbito de 0 a $29 \%^{9,10}$.

Assim, o objetivo desse estudo é analisar retrospectivamente os resultados da aplicação do tubo gástrico de grande curvatura isoperistáltico no tratamento cirúrgico paliativo em pacientes portadores de carcinoma avançado de esôfago torácico.

\section{MÉTODO}

No período de julho de 1992 a dezembro de 2001, 236 pacientes com carcinoma de esôfago foram atendidos no Serviço de Clínica Cirúrgica do Hospital e Maternidade Celso Pierro da Faculdade de Ciências Médicas da PUC-Campinas. Em 122 (51,6\%), realizou-se a ressecção e nos 114 pacientes restantes $(48,4 \%)$, por apresentarem tumores irressecáveis ou por não terem condições clínicas satisfatórias, se optou pela realização de tratamento paliativo. Assim, jejunostomia foi realizada em 11 pacientes, radioterapia exclusiva em 13 pacientes, prótese endoscópica em 37 e tubo gástrico isoperistáltico de grande curvatura em 53, este último, o objeto deste estudo.

O diagnóstico da afecção e seu estadiamento foi realizado por meio de endoscopia digestiva alta, tomografia computadorizada de tórax e abdômen e traqueobroncoscopia.

Em todos os pacientes foram realizadas também avaliação clínica e nutricional para demonstrar se os mesmos tinham condições de serem submetidos ao ato cirúrgico proposto.

Após avaliação completa pelos parâmetros acima relatados, os pacientes eram incluídos no protocolo proposto de tratamento paliativo e submetidos ao tratamento cirúrgico seguido por radioterapia.

Em todos os pacientes foi realizado o tubo gástrico isoperistáltico de grande curvatura e transposto por via retrosternal, como proposto por Postlethwait ${ }^{11}$ A técnica envolve a criação de um tubo de mais ou menos $3 \mathrm{~cm}$ de largura com preservação dos vasos gastroepiplóicos direitos. Para tanto, o estômago é dividido em uma linha paralela à grande curvatura, com o auxílio de sutura mecânica linear. Esta divisão se estende do fundo ao antro gástrico. $\mathrm{O}$ estômago seccionado lembra um "V", no qual a parte correspondente à pequena curvatura drena o esôfago distal em relação ao tumor. O tubo gástrico de grande curvatura é passado por via subesternal e anastomosado ao coto proximal do esôfago cervical, cuja extremidade distal é fechada por uma sutura em plano único pela técnica manual. Este procedimento é completado por uma jejunostomia que é retirada quando o paciente retorna a deglutir por via oral, entre o $10^{\circ}$ e $19^{\circ}$ dias do pós-operatório.

$\mathrm{Na}$ avaliação clínica pós-operatória procurouse avaliar as complicações imediatas e tardias, a qualidade de vida no que se refere à ingestão oral, a satisfação com a cirurgia e o tempo de sobrevida.

\section{RESULTADOS}

Dos pacientes estudados, $46(86,7 \%)$ eram do sexo masculino e sete $(13,3 \%)$ do feminino, com idade variável entre 46 e 76 anos, com média de 56,8 anos. Trinta e seis $(67,9 \%)$ pacientes eram tabagistas e etilistas, nove $(16,9 \%)$ somente etilistas e três $(5,6 \%)$ somente tabagistas. Em $28(52,8 \%)$ pacientes o carcinoma se situava no terço superior torácico do esôfago, em $16(30,1 \%)$ no terço médio torácico do esôfago e nos nove restantes $(16,9 \%)$ no terço inferior torácico.

A tomografia computadorizada de tórax evidenciou que o tumor em todos os pacientes infiltrava as estruturas do mediastino (T4): em $29(54,7 \%)$ a árvore traqueobrônquica, em 11 (20,9\%) a aorta, em $10(18,8 \%)$, a árvore traqueobrônquica concomitante com a aorta e em três $(5,6 \%)$ a coluna vertebral torácica. A traqueobroncoscopia confirmou infiltração da árvore respiratória em 29 pacientes $(54,7 \%)$. A tomografia computadorizada do abdome revelou metástase hepática em dois pacientes (3,7\%). A avaliação clínica evidenciou hipertensão arterial descompensada em 15 pacientes $(28,1 \%)$, diabetes descompensado em oito (15\%) e concomitância dessas afecções em nove restantes $(16,9 \%)$. Todos esses pacientes foram tratados adequadamente no pré-operatório. $\mathrm{Na}$ avaliação nutricional, 17 pacientes $(32,1 \%)$ apresentavam 10 a $20 \%$ de perda ponderal, tendo sido submetidos à suporte nutricional por meio de dieta 
enteral por sonda nasoentérica ou nutrição parenteral por 10 a 15 dias no pré-operatório. Este tempo foi baseado na avaliação clínica e laboratorial, realizado pelo grupo de Nutrição e Dietética do Hospital Celso Pierro da PUC-Campinas.

Cinco pacientes $(9,4 \%)$ faleceram no $3^{\circ}, 4^{\circ}$, $7^{\circ}, 10^{\circ}$ e $12^{\circ}$ dias de pós-operatório, três por infarto agudo do miocárdio e/ou embolia pulmonar, um por infecção pulmonar e o restante por peritonite conseqüente à deiscência da sutura mecânica do tubogástrico.

Oito pacientes (15\%) apresentaram infecção pulmonar diagnosticada no $4^{\circ}$ e $8^{\circ}$ dias de pósoperatório com boa evolução, com tratamento clínico em seis deles. Nos dois pacientes restantes houve evolução para empiema pleural. Quinze pacientes $(28,3 \%)$ apresentaram deiscência da anastomose esofagotubogástrico com fístula cervical, entre o $3^{\circ} \mathrm{e}$ $10^{\circ}$ dias de pós-operatório. O diagnóstico desta complicação foi clínico em 13 pacientes pela saída de secreção salivar ou gástrica pelo dreno da região cervical e em dois radiológico pelo extravasamento de contraste ao nível da anastomose.

$\mathrm{O}$ fechamento destas fístulas ocorreu entre o $11^{\circ}$ e o $19^{\circ}$ dias de pós-operatório, com boa evolução dos pacientes.

Onze destes pacientes (23\%) evoluíram com estenose da anastomose esofagotubogástrico cervical com diagnóstico realizado entre o $25^{\circ}$ e o $60^{\circ}$ dias de pós-operatório, tendo sido realizada dilatação endoscópica com sessões variáveis de três a 12 dilatações, com evolução regular até o tempo de seguimento.

O período de internação variou de oito a 26 dias, com média de 14,5 dias. Dos 48 pacientes estudados no pós-operatório, em 34 (70,8\%), a radioterapia foi completada integralmente com 5.000 cgys. Em seis pacientes $(12,5 \%)$, por apresentarem dor torácica intensa, disfagia e infecção pulmonar, a radioterapia foi interrompida. Nos oito restantes $(16,6 \%)$, este procedimento não foi realizado por opção dos pacientes.

Em relação à deglutição, dos 48 pacientes que tiveram alta hospitalar, $37(77,0 \%)$ apresentaram ingestão para sólidos ou pastoso, sem restrições e 11 $(23,0 \%)$ ingestão para dieta liquidopastosa. Estes últimos pacientes foram os que apresentaram estenose da anastomose e que mesmo com dilatações endoscópicas não conseguiram ter ingestões adequadas.
Em relação à satisfação com a cirurgia, com exceção dos 11 pacientes $(23,0 \%)$ que apresentaram estenose da anastomose, os $37(77,0 \%)$ demonstraram estarem satisfeitos com o procedimento, retornando, muitos deles, às suas atividades habituais.

Vinte e três pacientes faleceram pela doença entre o $6^{\circ}$ e o $13^{\circ}$ mês de pós-operatório, com média de 7,5 meses.

Quatorze pacientes estão sendo acompanhados no ambulatório, entre dois e 16 meses de pósoperatório, com evolução satisfatória.

Em 11 pacientes houve perda de seguimento.

\section{DISCUSSÃO}

A partir das idéias originais de Beck e Carrel ${ }^{12}$ e os bons resultados apresentados por Postlethwait ${ }^{11}$ e Speranzini et $a l^{13}$, passou-se a vislumbrar a possibilidade de submeter os portadores de neoplasia avançada de esôfago a procedimento cirúrgico diferente do que se vinha propondo até então. Adotouse o longo tubo gástrico confeccionado com a grande curvatura, isoperistáltico, anastomosado ao esôfago cervical por via retroesternal.

Este tipo de esofagoplastia obedece aos princípios básicos que orientam o cirurgião na escolha ideal de um substituto esofágico: comprimento adequado da víscera para evitar tensão, vascularização arterial e venosa satisfatória, e disposição anatômica que permita boa função digestiva, em um único tempo cirúrgico ${ }^{1,7,14}$. A longa sutura gástrica existente neste tipo de procedimento pela utilização de grampeador linear cortante favorece tanto o coto gástrico que ficará na cavidade abdominal como a sutura do tubo que será anastomosado ao esôfago cervical. Isto é realizado sem haver qualquer tipo de contaminação da ferida operatória. O único momento em que existe a possibilidade de contaminação neste procedimento cirúrgico é o da realização da anastomose entre o esôfago cervical e o tubo gástrico no pescoço.

Com a facilidade de utilização de grampeadores cirúrgicos a partir da década de 80 , a confecção do tubo gástrico de grande curvatura passou a ser procedimento simples, rápido e seguro ${ }^{7}$.

Com o intuito de preservar a maior quantidade possível de anastomoses entre o território da artéria gastroepiplóica direita e da artéria gastroepiplóica esquerda na confecção deste tubo gástrico, procedese à dissecção do ligamento gastro-cólico o mais próximo possível do cólon transverso. Este tipo de 
procedimento também é preconizado por Watanabe ${ }^{15}$, para evitar isquemia e desenvolvimento de fístulas pósoperatórias, principalmente quando há descontinuidade entre a arcada da artéria gastroepiplóica direita e esquerda na grande curvatura gástrica.

A ligadura da artéria gastroepiplóica esquerda foi feita ao nível de sua emergência junto á cauda do pâncreas. Os vasos breves foram todos ligados e a transição esôfago-gástrica dissecada com o intuito de realizar boa liberação do estômago. Desta maneira, consegue-se facilmente aplicar o grampeador linear cortante próximo do ângulo de Hiss, em direção paralela à grande curvatura no sentido do piloro. Obtém-se, assim, grande conduto gástrico com 2 a 3 $\mathrm{cm}$ de diâmetro e com sua extremidade fechada e irrigado pela artéria gastroepiplóica direita, após a liberação da mesma do ligamento gastro-cólico. Praticamente todo o comprimento do tubo de grande curvatura, assim obtido, é utilizado para atingir a região cervical por via retroesternal e sem tensão, permitindo ressecção da sua porção mais cranial, que é mais sujeita à isquemia.

A anastomose esôfago gástrica cervical é propensa à deiscência em uma incidência variável de 5 a $58, \%$, principalmente quando o tubo é posicionado retrosternalmente ${ }^{7,16-20}$. Entretanto, a fístula consequiente é controlada, na grande maioria das vezes com tratamento conservador, mas pode resultar em estenose no local da anastomose $\mathrm{e}^{7,8,20}$

Em nosso estudo, dos 15 pacientes $(28,3 \%)$ que apresentaram fístula anastomótica, em 11 houve necessidade de dilatação devido à estenose da anastomose. Alguns autores referem às vezes necessidade de reoperação quando ocorre esta complicação e muitas vezes sem sucesso ${ }^{20}$.

Outros autores têm demonstrado que a incidência de fístula da anastomose esofagogástrica cervical é duas vezes maior em pacientes não ressecados em relação aos ressecados, possivelmente, devido ao grupo não ressecado apresentar maior grau de desnutrição ${ }^{21}$.

Em relação à ingestão oral dos 48 pacientes que tiveram alta hospitalar, 37 (77\%) obtiveram ingestão oral para alimentos sólidos ou pastosos, dados estes similares a outros autores ${ }^{7,13,16,20}$. Orringer et al. ${ }^{6}$ avaliando 166 pacientes portadores de doenças benignas do esôfago, que necessitaram esofagectomia e gastroplastia, referem que $44, \%$ conseguiram ingerir dieta pastosa ou líquido-pastosa em até um ano de pós-operatório, necessitando de uma ou mais sessões de dilatação endoscópica. Provavelmente esta seja uma forma de manifestação tardia da isquemia da víscera transposta e do esôfago cervical dissecado com conseqüiente reação cicatricial e estenose ao nível da anastomose.

Uma significativa porcentagem de pacientes apresenta complicações cardiovasculares e respiratórias, presente no nosso estudo em $22,6 \%$ e similar a de outros ${ }^{7,20,22}$. Isto se deve à idade geralmente mais avançada desses pacientes, além dos mesmos, muitas vezes, apresentarem no pré-operatório alterações cardiovasculares, como foi demonstrado nos 12 pacientes da nossa série que apresentaram essas complicações e que apresentaram na avaliação préoperatória, hipertensão arterial descompensada. $\mathrm{O}$ tabagismo também é outro fator relevante nos pacientes que apresentam essas complicações, hábito este também presente nos nossos pacientes. Este dado também é observado por outros autores ${ }^{7,20}$.

No que se refere à mortalidade pós-operatória imediata, esta incidência pode variar de 0 a $42 \%$ 7,16,20,23,24. Certamente, esta variação se deve às diferenças de critérios na seleção pré-operatória. Desde que se realize uma avaliação clínica préoperatória bem adequada, principalmente no que concerne às funções cardiovasculares, pulmonares e nutricionais, a tendência é que a incidência de mortalidade seja menor. A freqüência de $9,4 \%$ de óbito no nosso estudo, embora não desprezível, é aceitável pelo perfil de doentes que operamos, além de que a maioria dos pacientes faleceram por complicações cardiovasculares e todos apresentavam hipertensão e/ou diabetes na avaliação préoperatória.

A sobrevida média de 7,5 meses, demonstrada nos 23 pacientes do nosso estudo que foram avaliados até o óbito conseqüente à doença, também é similar a séries de outros que utilizam este tipo de procedimento $7,13,20$. Este tempo é bem aceitável em vista do caráter paliativo e da doença avançada vigente.

Tem havido na literatura relatos quanto à maior sobrevida dos pacientes portadores de neoplasia avançada do esôfago, na medida em que se consegue paliação da disfagia a mais adequada possível, sendo os métodos cirúrgicos os que melhor restauram a capacidade de deglutição por proporcionar melhora nutricional, diminuição da possibilidade de aspiração com conseqüente broncopneumonias e o benefício emocional e psicológico proporcionado pela nova capacidade de deglutição adequada ${ }^{25,26}$. 
Desta maneira, pode-se concluir que o tubo gástrico isoperistáltico de grande curvatura parece oferecer melhora significativa da ingestão oral com qualidade de vida satisfatória e morbidade e morta- lidade aceitáveis, em vista do perfil de pacientes em que se atua. Assim sendo, credencia-se como bom método paliativo para o tratamento cirúrgico das neoplasias malignas do esôfago.

\begin{abstract}
Background: The objective of this study is to determine the beneficial of palliative esophageal bypass with isoperistaltic gastric tube in patients with unresectable esophagus carcinoma. Methods: We studied 53 patients with unresectable squamous cell carcinoma of the esophagus as showed by endoscopic, radiologic and clinical data. Most of the patients were male and the average age was 56,8 years. The operation performed was an isoperistaltic gastric tube transposed through the retrosternal space. Results: Twentyeight patients (52\%) developed one or more complications and the most frequent ones were leakage or stenosis of cervical anastomosis (15 patients - 28,3\%). Mortality rate was $9,4 \%$. Of the 48 patients that survived, 37 (77\%) refered relief of disphagia in the follow-up. The mean survival rate of 23 patients was 7,5 months (six to 13 months) and 14 patients are doing well after two to 16 months offollow-up. Conclusions: Isoperistaltic gastric tube has acceptable morbidity and mortality with relief of dysphagia in the majority of the patients.
\end{abstract}

Key words: Esophagus; Esophageal neoplasms; Palliative care.

\section{REFERÊNCIAS}

1. Pinotti HW, Felix VN, Raia AA. - Revisão e análise crítica das técnicas de restabelecimento do trânsito esofágico. An Paul Med Cir, 1978, 105: 1-28.

2. Mirra AP, Guida Filho B, Miziara JE. - Tratamento do câncer do esôfago torácico e abdominal: casuística de 103 casos operados. Rev Paul Med, 1976, 88: 118127.

3. Zilberstein B, Pinotti HW, Cecconello I, et al. - Câncer do esôfago. In: Pinotti HW (ed) - Tratado de clínica cirúrgica do aparelho digestivo. São Paulo. Atheneu, 1994, pp. 415-426.

4. Orel JJ, Vidmar SS, Hrabar BA - Intrathoracic gastric and jejunal bypass for palliation of nonresectable esophageal carcinoma. Int Surg, 1982, 67(2): 147-151.

5. Zilberstein B. - Esofagectomia cervico-abdominal por via transdiafragmática no tratamento cirúrgico do câncer do esôfago. Resultados e evolução a longo prazo. Dissertação (Livre-Docência). São Paulo. Universidade de São Paulo, 1988, 94 p.

6. Orringer MB, Marshall B, Stirling MC. - Transhiatal esophagectomy for benign and malignant disease. $\mathrm{J}$ Thorac Cardiovasc Surg, 1993, 105(2): 265-276.
7. Meniconi MTM. Estudo prospectivo da aplicação do tubo gástrico de grande curvatura, isoperistáltico, no tratamento da neoplasia avançada de esôfago. Análise de 50 casos. Dissertação (Doutorado). São Paulo. Universidade de São Paulo, 1997, 174 p.

8. Moura EGH - Tratamento paliativo do câncer do esôfago por endoprótese e tubo gástrico isoperistáltico. Dissertação (Doutorado). São Paulo. Universidade de São Paulo, 2000, 153 p.

9. Redd CE. - Comparison for different treatments for unresectable esophageal cancer. World J Surg, 1995, 19(6): 828-835.

10. Moreira LS, Coelho RC, Sadala RU. - The use of ethanol injection under endoscopic control to palliate dysphagia caused by esophagogastric cancer. Endoscopy, 1994, 26(3): 311-314.

11. Postlethwait RW. - Technique for isoperistaltic gastric tube for esophageal bypass. Ann Surg, 1979, 189(6): 673-676.

12. Beck C, Carrell A. - Demonstration of specimens illustrating a method of formation of a prethoracic esophagus. Illinois Med J, 1905, 7: 463-468.

13. Speranzini MB, Fujimura I, Pires PW. - Esofagoplastia com tubo gástrico isoperistáltico em derivação no 
tratamento do câncer do esôfago torácico: estudo de 13 casos. AMB Rev Assoc Med Bras, 1989, 35(3): 91-98.

14. Zilberstein B, Cecconello I, Pollara WM, Emprego do jejuno no restabelecimento do trânsito-esofágico, análise crítica das técnicas e dos resultados. In Pinotti HW - Condutas técnicas na cirurgia do esôfago. Cidade. Kronos, 1982, pp. 80.

15. Watanabe H. - The techniques to protect the anastomotic leakage of esophagogastrostomy at the neck. Nippon Geka Gakkai Zasshi, 1996, 97(6): 432-436.

16. Abe S, Tachibana M, Shimokawa T, et al. - Surgical treatment of advanced carcinoma of the esophagus. Surg Gynecol Obstet., 1989, 168(2): 115-120.

17. Paricio PP, Garcia Marcilla JA, Martinez de Haro L, et al. - Results of surgical treatment of epidermoid carcinoma of the thoracic esophagus. Surg Gynecol Obstet, 1993, 177(4): 398-404.

18. Zhang DW, Cheng GY, Huang GJ, et al. - Operable squamous esophageal cancer: current results from the East. World J Surg, 1994, 18(8): 347-354.

19. Saito T, Shigemitsu Y, Kinoshita T, et al. - Extended survival time for esophageal cancer patients treated with aggressive surgery and concurrent chemoradiation. Int Surg, 1994, 79(2): 93-97.

20. Alcantara PS, Spencer-Netto FA, Silva-Junior JF, et al. - Gastro-esophageal isoperistaltic bypass in the palliation of irresectable thoracic esophageal cancer. Int Surg, 1997, 82(3): 249-253.
21. Lorentz T, Fok M, Wong J. - Anastomotic leakage after resection and bypass for esophageal cancer: lessons learned from the past. World J Surg, 1989, 13(4): 472477.

22. Fan ST, Lau WY, Yip WC, et al. - Prediction of postoperative pulmonary complications in oesophagogastric cancer surgery. Br J Surg, 1987, 74(5): 408-410.

23. Mannell A, Becker PJ, Nissenbaum M - Bypass surgery for unresectable oesophageal cancer: early and late results in 124 cases. Br J Surg, 1988, 75(3): 283286.

24. Orringer MB. - Substernal gastric bypass of the excluded esophagus: results of an ill-advised operation. Surgery, 1984, 96(3): 467-470.

25. O'Hanlon DM, Harkin M, Karat D, et al. - Quality of life assessment in patients undergoing treatment for oesophageal carcinoma. Br J Surg, 1995, 82(12): 16821685.

26. Siegel HI, Laskin KJ, Dabezies MA, et al. - The effect of endoscopic laser therapy on survival in patients with squamous-cell carcinoma of the esophagus. Further experience. J Clin Gastroenterol, 1991, 13(2): 142146.

Endereço para correspondência:

Dr. José Luis Braga de Aquino

Rua Boaventura do Amaral 1250/10 and. Centro

13015-192 Campinas - SP 